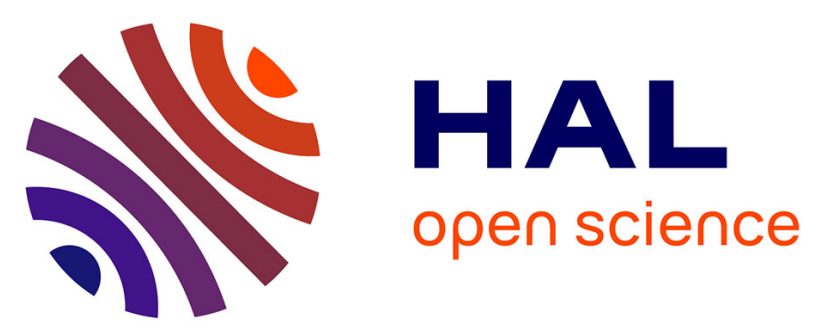

\title{
A novel 1-gram insect based device measuring visual motion along 5 optical directions
}

Frédéric L. Roubieu, Fabien Expert, Marc Boyron, Benoît-Jérémy Fuschlock, Stéphane Viollet, Franck Ruffier

\section{> To cite this version:}

Frédéric L. Roubieu, Fabien Expert, Marc Boyron, Benoît-Jérémy Fuschlock, Stéphane Viollet, et al.. A novel 1-gram insect based device measuring visual motion along 5 optical directions. Sensors, 2011 IEEE, Oct 2011, Limerick, Ireland. pp.687, 10.1109/ICSENS.2011.6127157 . hal-00716606

\section{HAL Id: hal-00716606 https://hal.science/hal-00716606}

Submitted on 10 Jul 2012

HAL is a multi-disciplinary open access archive for the deposit and dissemination of scientific research documents, whether they are published or not. The documents may come from teaching and research institutions in France or abroad, or from public or private research centers.
L'archive ouverte pluridisciplinaire HAL, est destinée au dépôt et à la diffusion de documents scientifiques de niveau recherche, publiés ou non, émanant des établissements d'enseignement et de recherche français ou étrangers, des laboratoires publics ou privés. 


\title{
A novel 1-gram insect based device measuring visual motion along 5 optical directions
}

\author{
Frédéric L. Roubieu*, Fabien Expert*, Marc Boyron*, Benoît-Jérémy Fuschlock*, Stéphane Viollet* and Franck Ruffier* \\ * Biorobotics dpt., Institute of Movement Sciences, CNRS \& Aix-Marseille Univ., France \\ Email: \{frederic.roubieu, fabien.expert, marc.boyron, stephane.viollet, franck.ruffier\}@ univmed.fr
}

\begin{abstract}
Autopilots for micro aerial vehicles (MAVs) with a maximum permissible avionic payload of only a few grams need lightweight, low-power sensors to be able to navigate safely when flying through unknown environments. To meet these demanding specifications, we developed a simple functional model for an Elementary Motion Detector (EMD) circuit based on the common housefly's visual system. During the last two decades, several insect-based visual motion sensors have been designed and implemented on various robots, and considerable improvements have been made in terms of their mass, size and power consumption. The new lightweight visual motion sensor presented here generates 5 simultaneous neighboring measurements of the 1-D angular speed of a natural scene within a measurement range of more than one decade $\left[25^{\circ} / \mathrm{s}, 350^{\circ} / \mathrm{s}\right]$. Using a new sensory fusion method consisting in computing the median value of the 5 local motion units, we ended up with a more robust, more accurate and more frequently refreshed measurement of the 1-D angular speed.
\end{abstract}

\section{INTRODUCTION}

MAV is a class of Unmanned Aerial Vehicles (UAVs) which can be used for the remote observation of hazardous environments without any risk to human life. MAVs have to be equipped with sensors and flight control devices enabling them to perform obstacle avoidance, terrain following and landing [1][2][3]. However, the MAVs of the future are expected to be reduced to insect-sized aircraft; this is why designing MAVs is an increasingly challenging task in which small size and low mass are essential requirements.

Flying insects are able to cross unknown environments without any sonars or laser range-finders: their visually guided performances depend mainly on optic flow (OF) sensing processes. The optic flow perceived by a moving agent (an animal, human or robot) is a vector field that gives the angular speed $\omega$ (magnitude in $\mathrm{rad} / \mathrm{s}$ ) at which any contrasting object in the environment is moving past the eyes. Onboard the fly, this angular speed is computed by a neuron called an Elementary Motion Detector (EMD), which is driven by at least two photoreceptors facing in different directions.

Based on studies on the fly's visual system previously conducted at our laboratory, several versions of fly-inspired 2-pixel motion sensors giving a single 1-D angular speed measurement were developed [4][5][6]. These visual motion sensors were therefore highly sensitive to measurement errors.

Here we present a new tiny visual motion sensor weighing only 1 gram (Fig. 1) based on 5 local motion units receiving visual inputs from a 6-pixel array. The accuracy and refresh rate of the angular speed measurements $\left(f_{\text {refresh }}\right)$ obtained with this visual motion sensor were dramatically improved by combining the 5 local motion units.

Section 2 gives a short description of the bio-inspired visual system and the principles underlying the individual 2-pixel "time of travel" scheme. Section 3 describes experiments performed on the visual motion sensor, which was tested indoors under natural light conditions. The results of these experiments are presented in section 4 .

\section{Description of The DEVICE}

The visual motion sensor designed and developed in this study is based on an off-the-shelf photosensor array (iC-LSC from iCHaus Company, http://www.ichaus.de) consisting of 2 rows of 6 photodiodes. A fixed-gain current amplifier is integrated into each photodiode. To sense a large number of contrasting objects, the photosensors in each column were paired to increase the signal/noise ratio by increasing the sensitive surface two-fold from $300 \mu m * 800 \mu m$ to $300 \mu m * 1600 \mu m$. This one-dimensional 6-pixel array was then mounted on a cheap, lightweight lens (Sparkfun SEN-00637, focal length $2 \mathrm{~mm}$, f-number 2.8) borrowed from a mobile telephone camera lens (Fig. 1).

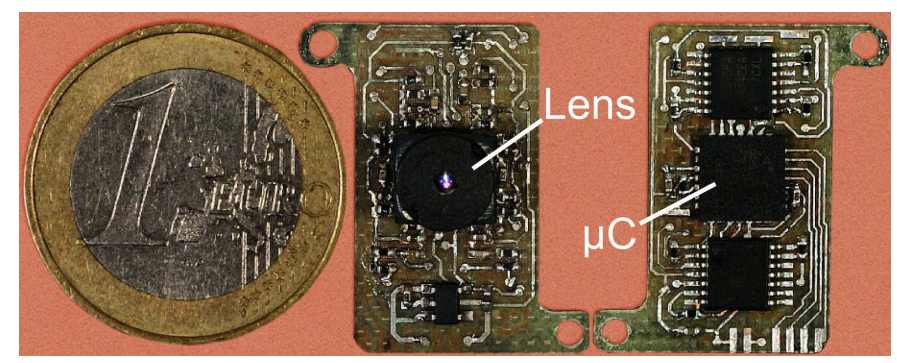

Fig. 1. Top view of the 1-gram microcontroller-based visual motion sensor (size: $23.3 \times 12.3 \mathrm{~mm}$ ) with its lens mounted on the one-dimensional 6-photosensor array, and bottom view of the PCB (thickness: $0.4 \mathrm{~mm}$ ) with its low-power dsPIC microcontroller $(\mu C)$.

The mass balance of our tiny device, including all the electronics, does not exceed 1 gram, which amounts to only $0.2 \mathrm{~g}$ per local motion unit (Tab. I). It is also a low-power visual motion sensor with a consumption of only $74 \mathrm{~mA}$ working at a voltage range from 3.5 to $5.5 \mathrm{~V}$.

The lens-photoreceptor system with which each local motion unit $i^{\text {th }}$ is equipped, assesses the angular speed $\omega_{i}(\mathrm{OF})$ of 


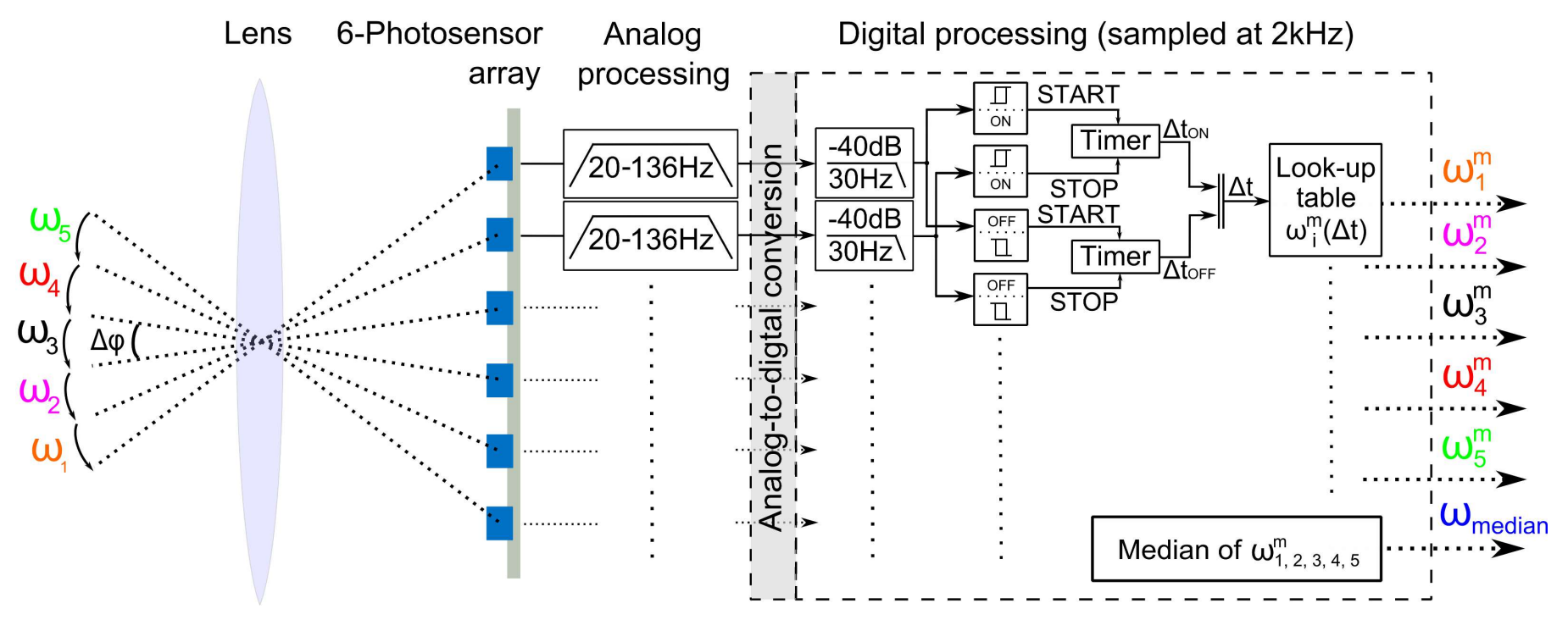

Fig. 2. Processing architecture of the visual motion sensor. The visual signals emitted by each photoreceptors are filtered through an analog bandpass filter and a second order fixed-point digital low-pass filter. Two neighboring signals are then thresholded. In parallel the "time of travel" $\Delta t$, elapsing between the two signals, is measured by a timer: $\Delta t_{O N}$ and $\Delta t_{O F F}$ are measured thanks to the ON and OFF contrast distinguishing processes [7]. These $\Delta t$ are used to generate the 1-D angular speed $\omega_{i}^{m}$ within the visual field of each 1-D local motion unit. Lastly, the 5 local motion units are combined to generate a robust and frequently refreshed 1-D median angular speed.

TABLE I

MASS BALANCE OF THE VISUAL MOTION SENSOR

\begin{tabular}{|c|c|}
\hline Parts & Mass $(\mathrm{g})$ \\
\hline PCB thickness 0.4mm, 3cm & 0.402 \\
\hline Lenslet Sparkfun SEN-00637 & 0.17 \\
\hline Lenslet-mount & 0.11 \\
\hline LSC iC-Haus retina & 0.13 \\
\hline microcontroller dsPIC 6x6 & 0.1 \\
\hline Electronic components & 0.162 \\
\hline Estimated total mass $=$ & 1.074 \\
\hline \multicolumn{2}{|c|}{ Real mass =0.98 g } \\
\hline Mass per 1-D local motion unit $<0.2 \mathrm{~g}$ \\
\hline
\end{tabular}

any contrasting object detected by 2 neighboring photosensor signals by measuring the delay $\Delta t_{i}$ defined as follows:

$$
\Delta t_{i}=\frac{\Delta \phi}{\omega_{i}}
$$

where $\Delta \phi$ is the inter-receptor angle (i.e., angle between two adjacent optical axes). The functional "time of travel" scheme used here consists of 6 processing steps giving the angular speed $\omega_{i}^{m}$ (Fig. 2):

- Step 1: Low-pass spatial filtering is achieved by defocusing the lens, thus giving each pixel in the array a Gaussian angular sensitivity function.

- Step 2: Analog bandpass filtering: high-pass filtering $\left(f_{c}=20 \mathrm{~Hz}\right)$ enhances the contrast information and eliminates the DC component of the photoreceptor signals. This step is followed by a first order low-pass filtering step with $f_{c}=136 \mathrm{~Hz}$.

- Step 3: Digitizing and filtering: second order fixed-point digital low-pass filtering $\left(f_{c}=30 H z\right)$ reduces any high frequency noise introduced by the artificial indoor lighting $(100 \mathrm{~Hz})$

- Step 4: Hysteresis thresholding is performed to distinguish between 'ON' and 'OFF' contrast transitions (i.e. dark-to-light and light-to-dark transitions, respectively) in each channel.

- Step 5: A time delay circuit is triggered by one channel and stopped by the neighboring channel. This function measures the delay time $\Delta t_{i}$ elapsing between similar ('ON' or 'OFF') transitions occurring in two adjacent photoreceptors.

- Step 6: Computing the 1-D angular speed within the visual field of the local motion unit by using a lookup table that transforms the delay $\Delta t_{i}$ into the measured angular speed $\omega_{i}^{m}$ by applying the equation 2 :

$$
\omega_{i}^{m}=\frac{\Delta \phi}{\Delta t_{i}}
$$

Our visual motion sensor generates 5 simultaneous local measurements of the 1-D angular speed of a natural scene within a measurement range of more than one decade $\left[25^{\circ} / s, 350^{\circ} / s\right]$. It also computes the median value $\omega_{\text {median }}$ of the 5 local motion units. The whole processing of the 5 local motion units and the calculation of the median value were carried out on a dsPIC33FJ128GP802 microcontroller working at a sampling frequency of $2 \mathrm{kHz}$. The microcontroller embedded onboard the visual motion sensor (Fig. 1) is connected to an external Bluetooth module, which conveys the data to a computer for analysis.

Like the angular sensitivity function (ASF) of a single fly photoreceptor, each photosensor features a Gaussian angular sensitivity function [8] (Fig. 3), which results in insects from 
the spatial convolution of the photoreceptor's diameter with the point spread function of the facet lenslet [9][10] and in our sensor, from the defocusing of the lenslet.

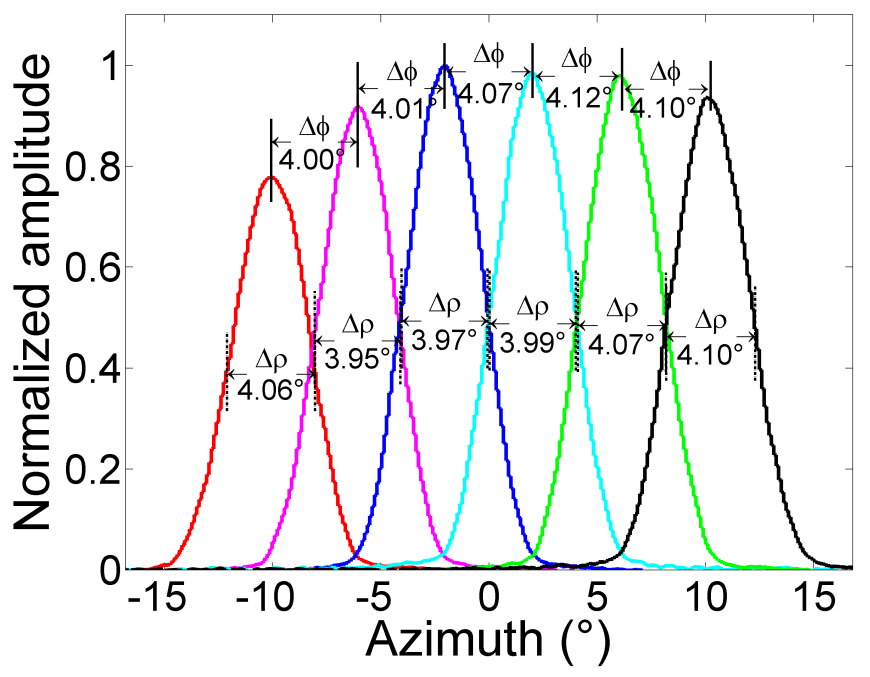

Fig. 3. Raw Angular Sensitivity Function (ASF) data obtained with the 6-photosensor array.

The ASF of the lens-photoreceptor system was assessed by slowly rotating the visual motion sensor after placing it $50 \mathrm{~cm}$ from a point light source. By defocusing the lens (i.e. by reducing the distance between the lens and the retina), we obtained the same Gaussian sensitivity profile as that of the fly. The full width at half height of the Gaussian curve (the acceptance angle) $\Delta \rho$ determines the cut-off frequency of the low-pass spatial filtering process (step 1), whereas $\Delta \phi$ determines the angular speed $(\omega)$ measurement range.

The defocusing process was adjusted to have an appropriate bell-shaped ASF projected onto the photosensor array as occurs in some diurnal insects [11] with:

$$
\Delta \phi=\Delta \rho
$$

The visual photoreceptor axes are separated by an angle $\Delta \phi=4^{\circ}$ and feature an acceptance angle $\Delta \rho=4^{\circ}$. The horizontal FOV of the visual motion sensor is $28.8^{\circ}$.

\section{EXPERIMENT}

The visual motion sensor was tested indoors with natural light at an illuminance of around 1500lux corresponding to the sunny daylight provided by a window.

The visual motion sensor was placed at an orthogonal distance $D_{h}$ from a printed strip of wallpaper showing a natural coloured scene. The printed strip was stretched between 2 actuated drums (see enclosed frame Fig. 4). The visual motion sensor was oriented at an arbitrary angle $\alpha$ between $V_{\text {wall }}$ and the main sensor axis (Fig. 4). The scene was therefore seen to move horizontally by the visual motion sensor at an angular speed $\omega_{\text {wall }}$ according to the equation 4 shown in Fig. 4.

With the orientation angle $\alpha$, we wanted to check that the measurements obtained with each local motion unit $i$ were in

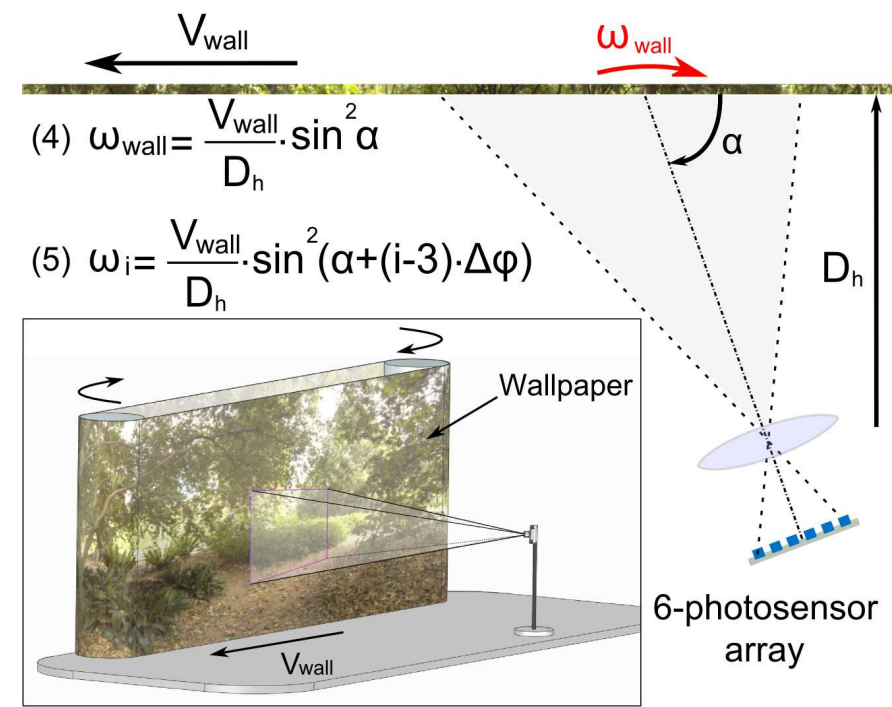

Fig. 4. The test bed for testing the visual motion device including the 5 local motion units.

line with the equation 5 shown in Fig. 4. The dynamic indoor responses were assessed and the combined output produced by the visual motion sensor at $\alpha=60^{\circ}$ with 2 different printed strips was recorded as follows:

- The first printed strip was decorated with a natural coloured scene (Fig. 5d)

- The second printed strip is decorated with an indoor coloured scene featuring a laboratory (Fig. 5h)

The visual motion sensor was placed at a distance $D_{h}=24 \mathrm{~cm}$ from the wall and the wallpaper was moved using a triangular speed law involving a series of velocity ramps with different slopes ranging between $27^{\circ} / \mathrm{s}$ and $230^{\circ} / \mathrm{s}$. The use of an arbitrary value $\left(60^{\circ}\right)$ for the orientation angle $\alpha$, enabled us to confirm that the output of one local motion unit depends on its optical direction according to equation 5, as shown in Fig. 4.

\section{RESUlTS}

The dynamic indoor responses of the visual motion sensor and the median output of the 5 local motion units were studied. To assess the performances of our new tiny visual motion sensor, the dynamic response of a single 2-pixel motion sensor (the main contributor to the median value computation) were compared with the median output in terms of the refresh rate and the standard deviation error $\left(S t d_{\text {error }}\right)$. The main contributor is the one among the 5 values which is most frequently used to calculate the median. The refresh rate was defined as the number of new motion measurements per second correponding to a new contrast transition detected by one pixel and then by the second pixel with any delay $\Delta t$ in the measurement range (the angular speed is in the $\left[25^{\circ} / \mathrm{s}, 350^{\circ} / \mathrm{s}\right]$ range). The $S t d_{\text {error }}$ corresponds to the dispersion of the data between the angular speed measured and the predicted value. 

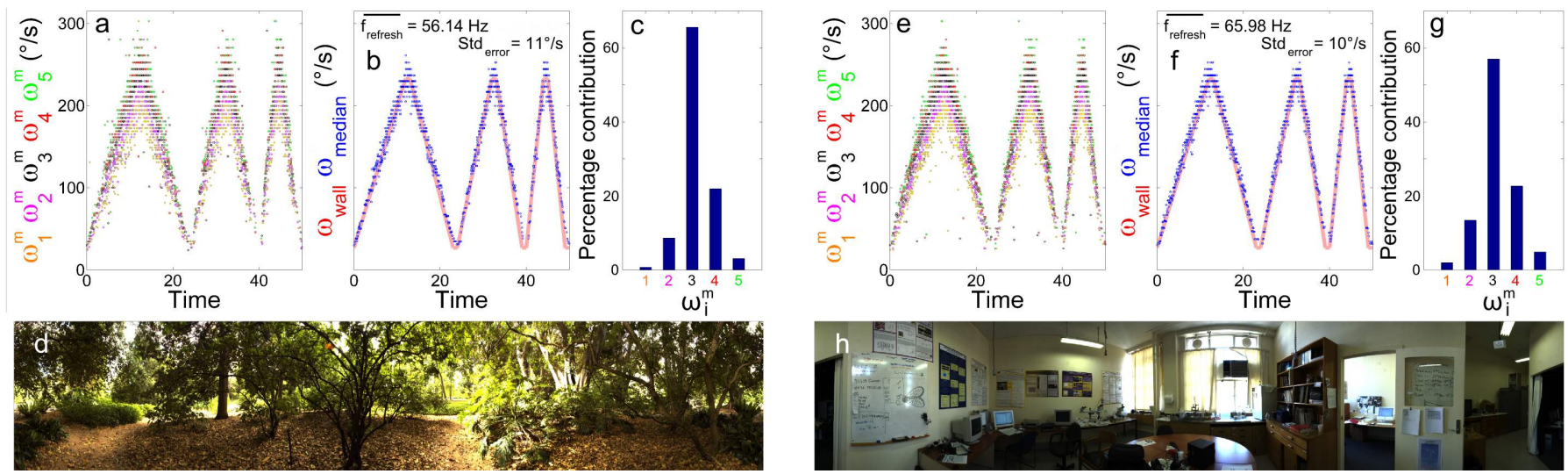

Fig. 5. Dynamic indoor responses of the visual motion sensor with 2 different printed belts. a and e) Dynamic indoor responses of each unit in the visual motion sensor to a series of velocity ramps induced in the printed belt, ranging from $27^{\circ} / \mathrm{s}$ to $230^{\circ} / \mathrm{s}$. b and f) Dynamic indoor responses in terms of the median values in comparison with those predicted by the main contributor. $\mathrm{c}$ and $\mathrm{g}$ ) Vertical bar graph showing which unit in the visual motion sensor was the main contributor to the median value computed, along with the standard deviation error (Std error $)$ and refresh-rate characteristics. $\mathrm{d}$ and $\mathrm{h}$ ) The natural coloured scene depicted on the printed belt (Fig. 4).

As expected from equation 5, which is shown in Fig. 4, the 5 local motion unit measurements are different because of the different orientations of the visual axes in the visual field (see Fig. 5a and e). Figs. 5c and g show that the main contributor to the median value at an orientation $\alpha=60^{\circ}$ is the $3^{\text {rd }}$ local motion unit. The median value faithfully follows the angular speed of the wall $\omega_{\text {wall }}$, giving a $S t d_{\text {error }}$ of only $11^{\circ} / \mathrm{s}$ with the printed belt showing a natural scene and $10^{\circ} / \mathrm{s}$ with the printed belt depicting a laboratory in comparison to the main contributor which is $19^{\circ} / \mathrm{s}$ and $22^{\circ} \mathrm{s}$ respectively. As shown in Figs. $5 \mathrm{~b}$ and $\mathrm{f}$, the refresh rate of the median value increased more than 4 -fold $(67 \mathrm{~Hz})$ in comparison with that of the main contributor $(15.7 \mathrm{~Hz})$ (Figs. 5c and g).

\section{CONCLUSion}

In this study, the dynamic characteristics of a new 1-gram bio-inspired visual motion sensor was tested indoors on a natural coloured scene under natural light conditions.

The results obtained here show that our new tiny visual motion sensor including 5 local motion units can generate 5 simultaneous measurements of the angular speed detected within their individual FOVs. In addition, the motion sensor yields a median value, which was found to be 1.7-fold more accurate and 4-fold more frequently refreshed than a single local motion unit's.

This visual motion sensor constitutes a good trade-off between the need for reliable motion sensors and the need for multiple small-sized electronic components. This visual motion sensor can be implemented on terrestrial and aerial vehicles and used in natural environments for obstacle avoidance, take-off, landing and speed control purposes.

\section{ACKNOWLEDGMENT}

We thank Dr. Jessica Blanc for correcting the English manuscript, R. Brinkworth and D. O'Carroll (Adelaide Uni., Australia) for kindly making their High Dynamic Range panoramic images available to us. This research was supported partly by CNRS (Life Science; Information and Engineering Science and Technology) Aix-Marseille University II, the French National Research Agency (ANR) (EVA project under ANR-ContInt grant number ANR608-CORD-007-04), and by European Commission via the CURVACE project. The CURVACE project acknowledges the financial support of the European Commission's Future and Emerging Programme for Research , under FET-Open grant number: 237940.

\section{REFERENCES}

[1] N. Franceschini, J. M. Pichon, C. Blanes, and J. M. Brady, "From insect vision to robot vision," Philosophical Transactions of the Royal Society B: Biological Sciences, vol. 337, pp. 283-294, 1992.

[2] F. Ruffier and N. Franceschini, "Optic flow regulation: the key to aircraft automatic guidance," Robotics and Autonomous Systems, vol. 50, no. 4, pp. 177 - 194, 2005.

[3] L. Kerhuel, S. Viollet, and N. Franceschini, "Steering by gazing: an efficient biomimetic control strategy for visually guided micro aerial vehicles," IEEE Transactions on Robotics, vol. 26, April 2010.

[4] M. Pudas, S. Viollet, F. Ruffier, A. Kruusing, S. Amic, S. Leppvuori, and N. Franceschini, "A miniature bio-inspired optic flow sensor based on low temperature co-fired ceramics (ltcc) technology," Sensors and Actuators A: Physical, vol. 133, no. 1, pp. 88 - 95, 2007.

[5] S. Viollet, F. Ruffier, T. Ray, M. Menouni, F. Aubepart, L. Kerhuel, and N. Franceschini, "Characteristics of three miniature bio-inspired optic flow sensors in natural environments," Sensor Technologies and Applications, International Conference on, vol. 0, pp. 51-55, 2010.

[6] F. Expert, S. Viollet, and R. F., "Outdoor field performances of insectbased visual motion sensors," Journal of Field Robotics, pp. 529-540, 2011.

[7] R. A. Franceschini, N. and A. L. Nestour, "Directionally selective motion detection by insect neurons," Facets of Vision, pp. 360-390, 1989.

[8] K. Götz, "Optomotorische untersuchung des visuellen systems einiger augenmutanten der fruchtfliege drosophila," Biological Cybernetics, vol. 2, pp. 77-92, 1964.

[9] N. Franceschini and K. Kirschfeld, "In vivo optical study of photoreceptor elements in the compond eye of drosophila," Biological Cybernetics, vol. 8, pp. 1-13, 1971 .

[10] D. G. Stavanga, "Angular and spectral sensitivity of fly photoreceptor. i. integrated facet lens and rhabdomere optics," Journal of Comparative Physiology A, vol. 189, pp. 1-17.

[11] M. F. Land, "Visual acuity in insects," Annual Review of Entomology, vol. 42, pp. 147-177, 1997. 